\title{
KIC 10526294: a slowly rotating B star with rotationally split, quasi-equally spaced gravity modes $\star, \star \star$ (Corrigendum)
}

\author{
P. I. Pápics ${ }^{1}$, E. Moravveji ${ }^{1, \star \star \star}$, C. Aerts ${ }^{1,2}$, A. Tkachenko ${ }^{1, \star \star \star \star}$, S. A. Triana ${ }^{1, \dagger}$, S. Bloemen ${ }^{1,2}$, and J. Southworth ${ }^{3}$ \\ 1 Instituut voor Sterrenkunde, KU Leuven, Celestijnenlaan 200D, 3001 Leuven, Belgium \\ e-mail: Peter.Papics@ster.kuleuven.be \\ 2 Department of Astrophysics, IMAPP, Radboud University Nijmegen, PO Box 9010, 6500 GL Nijmegen, The Netherlands \\ 3 Astrophysics Group, Keele University, Staffordshire, ST5 5BG, UK
}

A\&A 570, A8 (2014), DOI: 10.1051/0004-6361/201424094

Key words. asteroseismology - stars: variables: general - stars: early-type - stars: fundamental parameters - stars: oscillations stars: rotation - errata, addenda

An error occurred during the production process. An incorrect Fig. 12 was published. The correct Fig. 12 is published on the following page.

* Based on observations made with the William Herschel Telescope operated by the Isaac Newton Group on the island of La Palma at the Spanish Observatorio del Roque de los Muchachos of the Instituto de Astrofísica de Canarias.

$\star \star$ Appendices are available in electronic form at http://www . aanda.org

$\star \star \star$ Postdoctoral Fellow of the Belgian Science Policy Office (BELSPO), Belgium.

$\star \star \star \star$ Postdoctoral Fellow of the Fund for Scientific Research (FWO), Flanders, Belgium.

$\dagger$ Postdoctoral Fellow of the Fund for Scientific Research (FWO), Flanders, Belgium. 
A\&A 570, C4 (2014)
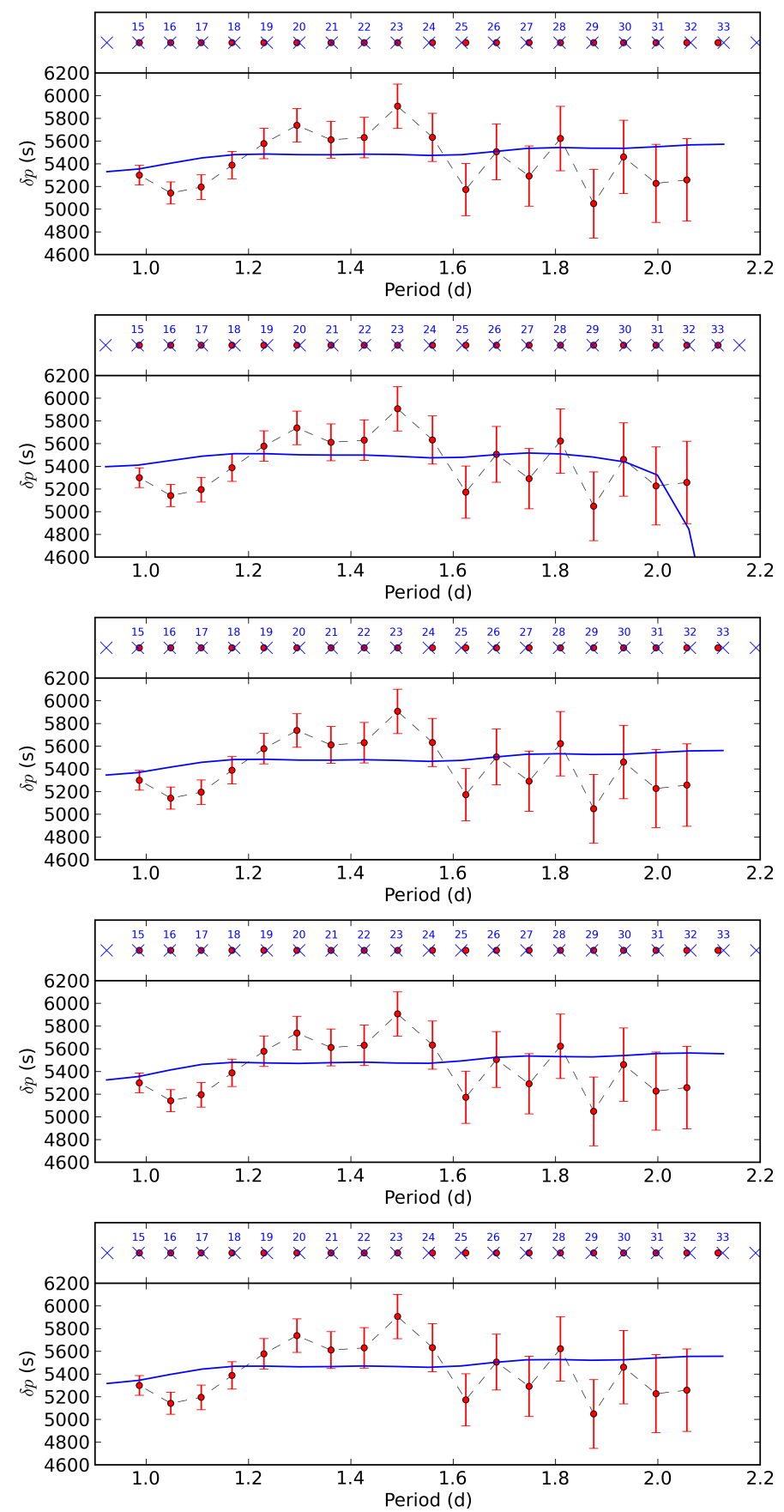

Fig. 12. Five best fit models (from top to bottom, following the order in Table 6) using frequencies from MESA/GYRE. Upper panels: observed peaks (red circles, the error bars are smaller than the symbol size) versus the theoretical frequencies of the $\ell=1$ gravity modes (blue crosses, with the corresponding $n$ value printed above). Lower panels: individual spacing values between the members of the observed period series (red filled circles) and the $\delta p(p)$ function from the model (blue solid line). 